\title{
Quantum regime of free electron lasers starting from noise
}

\author{
R. Bonifacio, ${ }^{1}$ N. Piovella, ${ }^{1,2}$ G. R. M. Robb,${ }^{3}$ and A. Schiavi ${ }^{4}$ \\ ${ }^{1}$ INFN-Sezione di Milano, Via Celoria 16, I-20133 Milano, Italy \\ ${ }^{2}$ Dipartimento di Fisica, Università Degli Studi di Milano, Via Celoria 16, I-20133 Milano, Italy \\ ${ }^{3}$ SUPA, Department of Physics, University of Strathclyde, Glasgow, G4 ONG, Scotland, United Kingdom \\ ${ }^{4}$ Dipartimento di Energetica, Università di Roma "La Sapienza" and INFN, Via Scarpa 14, I-00161 Roma, Italy
}

(Received 23 June 2006; published 5 September 2006)

\begin{abstract}
We investigate the quantum regime of a high-gain free-electron laser starting from noise. In the first part, we neglect the radiation propagation and we formulate a quantum linear theory of the $\mathrm{N}$-particle freeelectron laser Hamiltonian model, quantizing both the radiation field and the electron motion. Quantum effects such as frequency shift, line narrowing, quantum limitation for bunching and energy spread, and minimum uncertainty states are described. Using a second-quantization formalism, we demonstrate quantum entanglement between the recoiling electrons and the radiation field. In the second part, we describe the field classically but we include propagation effects (i.e. slippage) and we demonstrate the novel regime of quantum SASE with high temporal coherence and discrete spectrum. Furthermore, we describe "quantum purification" of SASE: the classical chaotic spiking behavior disappears and the spectrum becomes a series of discrete very narrow lines which correspond to transitions between discrete momentum eigenstates (which originate high temporal coherence).
\end{abstract}

DOI: 10.1103/PhysRevSTAB.9.090701

PACS numbers: 41.60.Cr, 42.50.Fx

\section{INTRODUCTION}

The quantum dynamics of an free-electron laser (FEL) is determined by a "quantum FEL parameter," $\bar{\rho}[1,2]$, defined in terms of the classical $\rho$ parameter, introduced by Bonifacio, Pellegrini, and Narducci [3] as

$$
\bar{\rho}=\rho \frac{m c \gamma_{R}}{\hbar k},
$$

which represents the ratio between the classical momentum spread and the one-photon recoil momentum. The classical limit is recovered only when this parameter is much larger than 1 . In this classical limit, the FEL dynamics are independent of $\bar{\rho}$ and the FEL equations can be universally scaled with no free parameters. On the contrary, when $\bar{\rho} \leq 1$, one has strong quantum effects and the FEL dynamics depend explicitly on $\bar{\rho}$.

Previous quantum treatments [1,2] of the high-gain exponential regime of FELs have been presented in the past years. However, the analysis used in these quantum models defines noncommuting particle collective operators without the necessary symmetrization. As a consequence, the results produced using these models are incorrect.

In the first part of the paper, both the electron motion and the radiation field are quantized, using a multiparticle Hamiltonian approach. We show the existence of a general uncertainty principle relating momentum spread and bunching, which implies that the maximum bunching is limited by energy spread. We define a minimum uncertainty state, which, for small fluctuations, reduces to a Gaussian packet. Finally, the multiparticle approach is compared with a second-quantization approach in terms of momentum states occupation operators. Photon statistics and quantum entanglement in an FEL, starting from vacuum fluctuations, are derived and discussed in the steady-state linear regime.

In the second part, we describe classically the field and we include propagation/slippage effects using a selfconsistent system of Schrödinger-Maxwell equations. This allows us to provide a quantum description of selfamplified superradiant emission (SASE). The SASE mode for a FEL is made up of three basic ingredients: high-gain, propagation or "slippage" effects, and start-up from noise [4]. The classical steady-state high-gain regime of FELs, with universal scaling and the introduction of the $\rho$ parameter, was analyzed in [3], where the possibility of operating an FEL in the SASE regime was suggested. Other treatments assume that SASE is just steady-state instability starting from noise [5,6]. This approach does not give the correct temporal structure and spectrum of SASE radiation as described in [4]. As a matter of fact, in Refs. [7-9] it has been shown that, due to propagation, there exists not only the steady-state instability of [3], but also a superradiant instability, with peak intensity proportional to $n^{2}$, where $n$ is the electron density. This superradiant instability, entirely due to slippage, is the heart of SASE, so that all the treatments which claim to describe SASE without this propagation induced instability are incomplete.

As shown in [4], a SASE FEL radiates a random series of superradiant spikes because, roughly speaking, at short wavelength, the electron bunch contains many cooperation lengths which radiate randomly and independently from one another. The number of spikes in the high-gain regime corresponds approximately to the number of cooperation lengths in the electron bunch (precisely $L_{b} / 2 \pi L_{c}$ ). The final result is an almost chaotic temporal pulse structure 
with a broad spectral width, unless $L_{b} \leq 2 \pi L_{c}$ [4]. Hence, classical SASE has one drawback with regard to its application as a useful source of short-wavelength coherent light: when $L_{b} \gg 2 \pi L_{c}$ its temporal coherence is very poor due to the noisy spectrum.

Here we propose a novel method for producing coherent short-wavelength radiation with SASE by adding new features to a previous quantum treatment [10]. We show that when $\bar{\rho} \gg 1$ the SASE FEL behaves classically, i.e., in agreement with the SASE classical model. In this limit we demonstrate analytically the classical limit: the equation for the quantum Wigner function associated to the Schrödinger-like equation reduces to the classical Vlasov equation. However, when $\bar{\rho} \leq 1$, we obtain a quantum regime with features completely different from those of the classical regime and to which we shall refer as quantum SASE. A surprising feature of this regime is the phenomenon of "quantum purification," in which the chaotic spectrum of classical SASE is replaced by a completely different coherent spectrum, as if the system would be driven by a coherent seed. More specifically, in the quantum regime one has a set of discrete narrow lines equally spaced due to transition between discrete momentum states. Increasing $\bar{\rho}$, the distance between the lines decreases and their width increases. The classical continuous noisy spectrum is recovered when, for $\bar{\rho}>0.4$, the lines overlap.

\section{HAMILTONIAN MODEL}

We start from the FEL Hamiltonian for $N$ electrons interacting with a single mode of radiation [1]:

$$
H=\sum_{j=1}^{N}\left[\frac{p_{j}^{2}}{2 \bar{\rho}}+i g\left(a^{\dagger} e^{-i \theta_{j}}-a e^{i \theta_{j}}\right)\right]-\delta a^{\dagger} a,
$$

where $\theta_{j}=\left(k+k_{w}\right) z-c k t_{j}-\delta \bar{z}$ and $p_{j}=m c\left(\gamma_{j}-\right.$ $\left.\gamma_{0}\right) / \hbar\left(k+k_{w}\right)$ are position and momentum operators of the $j$ th electron, with $\left[\theta_{i}, p_{j}\right]=i \delta_{i j}, a$ is the annihilation operator of the radiation field, with $\left[a, a^{\dagger}\right]=1, g=$ $\sqrt{\bar{\rho} / N}, \bar{z}=z / L_{g}, L_{g}=\lambda_{w} / 4 \pi \rho$ is the gain length, $\delta=$ $\left(\gamma_{r}-\gamma_{0}\right) / \rho \gamma_{0} \quad$ is the detuning, $\rho=\left(1 / \gamma_{r}\right) \times$ $\left(a_{w} \omega_{p} / 4 c k_{w}\right)^{2 / 3}$ is the classical FEL parameter, $\omega_{p}=$ $\sqrt{e^{2} n / m \epsilon_{0}}$ is the plasma frequency, $\gamma_{r}=$ $\sqrt{k\left(1+a_{w}^{2}\right) / 2 k_{w}}$ is the resonant energy (in $m c^{2}$ units), and $a_{w}$ is the undulator parameter. We observe that the dynamics depends on the single quantum FEL parameter $\bar{\rho}$ defined in (1). From the Hamiltonian (2), we derive the following Heisenberg evolution equations:

$$
\begin{gathered}
\frac{d \theta_{j}}{d \bar{z}}=\frac{p_{j}}{\bar{\rho}} \\
\frac{d p_{j}}{d \bar{z}}=-g\left(a e^{i \theta_{j}}+a^{\dagger} e^{-i \theta_{j}}\right)
\end{gathered}
$$

$$
\frac{d a}{d \bar{z}}=g \sum_{j=1}^{N} e^{-i \theta_{j}}+i \delta a .
$$

A constant of motion, which represents the total momentum in dimensionless units, is given by

$$
\sum_{j=1}^{N} p_{j}+a^{\dagger} a=\text { const. }
$$

Let us introduce the following electron collective operators:

$$
\begin{gathered}
B=\frac{1}{\sqrt{N}} \sum_{j=1}^{N} e^{-i \theta_{j}} \\
P=\frac{1}{\sqrt{N}} \sum_{j=1}^{N}\left(\frac{p_{j} e^{-i \theta_{j}}+e^{-i \theta_{j}} p_{j}}{2}\right),
\end{gathered}
$$

where $B$ is the bunching and $P$ is the symmetrized momentum bunching. This symmetrization is fundamental whenever one is dealing with products of noncommuting operators, i.e., $\left[e^{-i \theta_{j}}, p_{k}\right]=\delta_{j k} e^{-i \theta_{j}}$.

We consider $a, p_{j}$, and $\sum_{j} e^{-i \theta_{j}}$ as fluctuation operators, i.e., the initial states for the electrons and the field is such that $\langle a\rangle_{0}=\left\langle p_{j}\right\rangle_{0}=\sum_{j}\left\langle e^{-i \theta_{j}}\right\rangle_{0}=0$. Writing the Heisenberg equations of motion and neglecting the highorder quantities

$$
\frac{1}{\sqrt{N}} \sum_{j}\left(p_{j} e^{-i \theta_{j}} p_{j}\right), \quad a^{\dagger} \frac{1}{N} \sum_{j} e^{-2 i \theta_{j}},
$$

we obtain the following equations for the linear regime:

$$
\begin{gathered}
\frac{d B}{d \bar{z}}=-\frac{i}{\bar{\rho}} P \\
\frac{d P}{d \bar{z}}=-\frac{i}{4 \bar{\rho}} B-\sqrt{\bar{\rho}} a \\
\frac{d a}{d \bar{z}}=\sqrt{\bar{\rho}} B+i \delta a .
\end{gathered}
$$

The quantum correction to the classical description [3] is given by the term $-i B / 4 \bar{\rho}$ in the equation for $P$. Looking for solutions of the linear system (10)-(12) of the form $B(\bar{z})=B_{0} \exp (i \lambda \bar{z})$, we obtain the cubic characteristic equation

$$
(\lambda-\delta)\left(\lambda^{2}-\frac{1}{4 \bar{\rho}^{2}}\right)+1=0 .
$$

Notice that this dispersion relation coincides with that of a classical FEL with an initial energy spread with a square distribution and width $1 / 2 \bar{\rho}$, i.e., this extra term represents the intrinsic quantum momentum spread which, in dimensional units, becomes $\hbar k / 2$. In [1,2] the linear approximation has been carried out without properly symmetrizing 
the momentum bunching operator, defined in (8). In fact, in [1] the authors define the momentum bunching as $P_{1}=$ $(1 / \sqrt{N}) \sum_{j} \exp \left(-i \theta_{j}\right) p_{j}$ and neglect in the linear approximation the high-order term $\sum_{j} \exp \left(-i \theta_{j}\right) p_{j}^{2}$, leading to the equation $d P_{1} / d \bar{z}=-a$ and to the classical cubic equation $\lambda^{2}(\lambda-\delta)+1=0$ [3], which can be obtained from Eq. (13) in the limit $\bar{\rho} \gg 1$. In [2], the authors define the momentum bunching as $P_{2}=(1 / \sqrt{N}) \sum_{j} p_{j} \exp \left(-i \theta_{j}\right)$ without symmetrizing. Neglecting the high-order term $\sum_{j} p_{j}^{2} \exp \left(-i \theta_{j}\right)$, they obtain the following cubic equation [see Eq. (27) in [2]]:

$$
\lambda^{3}-(\delta+q) \lambda^{2}+\left(\delta q+q^{2} / 4\right) \lambda+1-\delta q^{2} / 4=0,
$$

where $q=1 / \bar{\rho}$. However, defining $\lambda^{\prime}=\lambda-q / 2$ and $\delta^{\prime}=\delta-q / 2$, Eq. (14) becomes formally identical to the usual classical cubic $\lambda^{\prime 2}\left(\lambda^{\prime}-\delta^{\prime}\right)+1=0$, just redefining the detuning parameter. As a consequence, the analysis of the quantum corrections discussed in [2], in which the resonance is assumed for $\delta=0$, instead of $\delta=q / 2=$ $1 / 2 \bar{\rho}$, is not correct. As a matter of fact the cubic equation which describes correctly the quantum behavior is not given by Eq. (14), but by Eq. (13), which has been obtained using the correct symmetrization of the collective operator, as given by Eq. (8). The features of the solution of the cubic equation (13) are shown in Fig. 1. When $\bar{\rho} \leq 1$ [Figs. 1(b)-1(f)], the resonance occurs at $\delta=1 /(2 \bar{\rho})$, with full width equal to $4 \sqrt{\bar{\rho}}$ and peak value $\operatorname{Im} \lambda=\sqrt{\bar{\rho}}$. Note that the field and the bunching grow exponentially as $\exp (\sqrt{\bar{\rho}} \bar{z})=\exp \left(z / L_{g}^{\prime}\right)$, where $L_{g}^{\prime}=L_{g} / \sqrt{\bar{\rho}}=$ $\lambda_{w} /(4 \pi \rho \sqrt{\rho})$ is the quantum gain length.

Hence, in the quantum regime $\bar{\rho}<1$, the resonance condition is $m c\left(\gamma_{r}-\gamma_{0}\right)=\hbar k / 2$ (i.e. $\delta=1 / 2 \bar{\rho}$ our dimensionless variables), the gain length increases and the gain bandwidth narrows as the square root of the quantum FEL parameter $\bar{\rho}$. On the contrary, if one uses the cubic

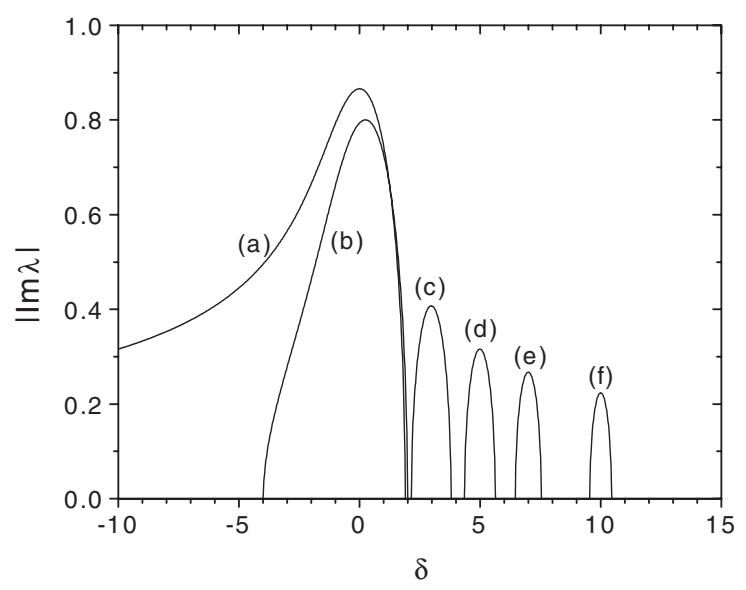

FIG. 1. Imaginary part of the complex root of the cubic Eq. (13) vs $\delta$ for $1 / 2 \bar{\rho}$ equal to 0 (a), 0.5 (b), 3 (c), 5 (d), 7 (e), and 10 (f).
(14) of [2], one would obtain Fig. 1(a) with the correct shift of the resonance to the right but with all the other properties of the quantum solution missing.

\section{UNCERTAINTY RELATIONS}

We now derive, from first principles, very general limitations for bunching and energy spread. The phase operator $\theta$, defined in the $(0,2 \pi]$ space, and the canonically conjugate momentum $p=-i \partial / \partial \theta$, satisfy the commutation rule $[\theta, p]=i$. These two variables can be interpreted also as azimuthal angle about the $z$ axis and $z$-component of the orbital momentum $L_{z}=p$, so that the momentum $p$ (in units of $\hbar k$ ) has discrete eigenvalues $n=0, \pm 1, \ldots$ and normalized eigenfunctions $(1 / \sqrt{2 \pi}) \exp (\operatorname{in} \theta)$. As it is well known [11], assuming these discrete eigenstates, one cannot conclude that the commutation rule implies the uncertainty relation $\Delta \theta \Delta p \geq 1 / 2$. However, other uncertainty relations can be obtained using the periodic operators $\cos \theta$ and $\sin \theta$, with commutation rules with $p$ given by $[\sin \theta, p]=i \cos \theta$ and $[\cos \theta, p]=-i \sin \theta$. Therefore, from the general uncertainty relations, one can deduce the following inequalities [11]:

$$
\begin{aligned}
& \Delta p \Delta \sin \theta=(1 / 2)|\langle\cos \theta\rangle| \\
& \Delta p \Delta \cos \theta=(1 / 2)|\langle\sin \theta\rangle|
\end{aligned}
$$

which can be combined in the single symmetrical relation:

$$
(\Delta p)^{2}\left[(\Delta \cos \theta)^{2}+(\Delta \sin \theta)^{2}\right] \geq \frac{1}{4}\left(\langle\cos \theta\rangle^{2}+\langle\sin \theta\rangle^{2}\right) .
$$

Defining the bunching $b=\langle\exp (-i \theta)\rangle=\langle\cos \theta\rangle-$ $i\langle\sin \theta\rangle$, Eq. (17) provides the following uncertainty relation between the momentum spread $\Delta p$ (in units of $\hbar k$ ) and the bunching:

$$
\Delta p \geq \frac{|b|}{2 \sqrt{1-|b|^{2}}}
$$

which can be written also as

$$
|b| \leq \frac{\Delta p}{\sqrt{\Delta p^{2}+1 / 4}} .
$$

The inequalities (18) and (19) are intrinsically quantum mechanical relations between bunching and energy spread and their validity is independent of the FEL dynamics.

Relation (19) set an upper limit to the maximum bunching obtainable in FELs, and states that $|b|$ can be near unity only when $\Delta p \gg 1 / 2$, i.e., when the momentum spread is much larger than $\hbar k / 2$. In the case in which one can assume $\Delta \theta \ll 1$, one has $|b|^{2} \approx 1-(\Delta \theta)^{2}$ and the relation (18) reduces to the usual Heisenberg uncertainty principle $\Delta \theta \Delta p \geq 1 / 2$.

We now introduce a minimum uncertainty state. It has been demonstrated [12] that there is no state that allows the symmetrical uncertainty relation Eq. (17) to reach its mini- 
mum value. However, there exist states that minimize one of the two uncertainty relations (15) and (16). These minimum uncertainty states are solutions of the equation $[11,12]$

$$
\left(\frac{\partial}{\partial \theta}+\gamma \sin \theta\right) \psi_{\lambda}(\theta)=i \lambda \psi_{\lambda}(\theta),
$$

which have the form

$$
\psi_{\lambda}(\theta)=G e^{-2 \gamma \sin ^{2}(\theta / 2)+i \lambda \theta} .
$$

Because $\psi_{\lambda}(\theta)$ must be a single valued function, then $\lambda=$ $m=\langle p\rangle,\langle\sin \theta\rangle=0$, and the normalization constant $G$ is given by $G^{-2}=\int_{0}^{2 \pi} d \theta e^{2 \gamma \cos \theta}=2 \pi I_{0}(2 \gamma)$, where $I_{n}$ is the modified Bessel function of order $n$. States (21) minimize the first uncertainty relation (15) and describe states with a nonzero energy spread. In fact, they reduce, for $\gamma=$ 0 , to the eigenstates $(1 / \sqrt{2 \pi}) \exp (\operatorname{im} \theta)$ of $p$, whereas for large values of $\gamma, \psi_{m} \approx(\gamma / \pi)^{1 / 4} \exp \left(-\gamma \theta^{2} / 2+i m \theta\right)$, i.e. the minimum uncertainty state becomes a Gaussian wave packet with $\Delta \theta=1 / \sqrt{2 \gamma}$ and $\Delta p=\sqrt{\gamma / 2}$, such that $\Delta \theta \Delta p=1 / 2$. In general, $\Delta p=$ $\sqrt{(\gamma / 2)\left[I_{1}(2 \gamma) / I_{0}(2 \gamma)\right]}[11]$. These states, originally introduced by Jackiw [12] to describe the phase of the photon could be useful to describe the energy spread in the quantum description of FELs.

\section{QUANTUM FIELD DESCRIPTION}

An alternative description to the $N$-particle Hamiltonian model can be formulated in the second-quantization formalism, treating the electrons as noninteracting bosons $[13,14]$. In this formulation, the $N$ particles are described by a matter-field operator $\hat{\Psi}(\theta, \bar{z})$ obeying the bosonic equal-time commutation relation

$$
\left[\hat{\Psi}(\theta), \hat{\Psi}(\theta)^{\dagger}\right]=\delta\left(\theta-\theta^{\prime}\right)
$$

and the normalization condition

$$
\int_{0}^{2 \pi} \hat{\Psi}(\theta)^{\dagger} \hat{\Psi}(\theta)=\hat{N}
$$

In this formulation, the second-quantized Hamiltonian is

$$
\hat{H}=\int_{0}^{2 \pi} \hat{\Psi}(\theta)^{\dagger} H\left(\theta,-i \frac{\partial}{\partial \theta}, a, a^{\dagger}\right) \hat{\Psi}(\theta),
$$

where $H$ is the single-particle Hamiltonian defined in (2). The Heisenberg equation for $\hat{\Psi}(\theta, \bar{z})$ and $a$ are

$$
\begin{gathered}
i \frac{\partial \hat{\Psi}}{\partial \bar{z}}=[\hat{\Psi}, \hat{H}]=-\frac{1}{2 \bar{\rho}} \frac{\partial^{2} \hat{\Psi}}{\partial \theta^{2}}+i g\left(a^{\dagger} e^{-i \theta}-a e^{i \theta}\right) \hat{\Psi} \\
\frac{d a}{d \bar{z}}=-i[a, \hat{H}]=g \int_{0}^{2 \pi} d \theta \hat{\Psi}(\theta)^{\dagger} e^{-i \theta} \hat{\Psi}(\theta)+i \delta a .
\end{gathered}
$$

Then, expanding the matter-wave field in the momentum basis,

$$
\hat{\Psi}(\theta)=\sum_{n=-\infty}^{+\infty} c_{n} u_{n}(\theta)
$$

where $u_{n}=(1 / \sqrt{2 \pi}) \exp (\operatorname{im} \theta)$ are the eigenfunctions of $p$ with eigenvalue $n$ and $c_{n}$ are the annihilation operators for the state with eigenvalue $n$, with $\left[c_{n}, c_{m}^{\dagger}\right]=\delta_{n, m}$. Then, using (27), Eqs. (25) and (26) become

$$
\begin{gathered}
\frac{d c_{n}}{d \bar{z}}=-i \frac{n^{2}}{2 \bar{\rho}} c_{n}+g\left(a^{\dagger} c_{n+1}-a c_{n-1}\right) \\
\frac{d a}{d \bar{z}}=g \sum_{n=-\infty}^{+\infty} c_{n-1}^{\dagger} c_{n}+i \delta a .
\end{gathered}
$$

The quantum expression for the bunching parameter appearing in the right-hand side of Eq. (29),

$$
b=\frac{1}{N} \sum_{n=-\infty}^{+\infty} c_{n-1}^{\dagger} c_{n}
$$

(where $N=\langle\hat{N}\rangle$ ), shows that electron bunching involves a coherent superposition of different momentum states. The semiclassical regime of Eqs. (28) and (29), in which $a$ and $c_{n}$ are treated as classical functions, has been investigated in [15]. A fully quantum treatment of the linear regime of Eqs. (28) and (29) has been given in [14], considering the equilibrium state with no photons and all the electrons in the state with $n=0$ (i.e. $\langle a\rangle_{0}=0$ and $\left\langle c_{0}^{\dagger} c_{0}\right\rangle_{0}=N$ ). Then, considering $c_{1}, c_{-1}$, and $a$ as fluctuation operators, we obtain the same quantum linear equations (10)-(12), in which the bunching and the momentum bunching operators are defined as $B=c_{1}+c_{-1}^{\dagger}$ and $P=c_{1}-c_{-1}^{\dagger}$. In this description the electrons have initially a definite value of momentum (i.e. $p=0$ ), so that they are delocalized in position. The dynamics of the system is that of three parametric coupled harmonic oscillators, $a_{1}=c_{-1}, a_{2}=c_{1}$, and $a_{3}=a$, which obey commutation rules $\left[a_{i}, a_{j}\right]=0$ and $\left[a_{i}, a_{j}^{\dagger}\right]=\delta_{i j}$ for $i, j=1,2,3$. Starting from the vacuum state of the three modes, it has been demonstrated [14] that the state at $\bar{z}$ is

$$
\begin{aligned}
|\Psi(\bar{z})\rangle= & \frac{1}{\sqrt{1+\left\langle n_{1}\right\rangle}} \sum_{m, n=0}^{\infty} \alpha_{1}^{m}(\bar{z}) \alpha_{2}^{n}(\bar{z}) \sqrt{\frac{(m+n) !}{m ! n !}} \\
& \times|m+n, n, m\rangle,
\end{aligned}
$$

where $\left|\alpha_{1,2}\right|^{2}=\left\langle n_{3,2}\right\rangle /\left(1+\left\langle n_{1}\right\rangle\right)$, and $\left\langle n_{i}\right\rangle=\left\langle a_{i}^{\dagger} a_{i}\right\rangle(i=$ $1,2,3)$ are the average occupation numbers. The state $n_{1}$ refers to electrons with negative recoil (decelerating), $n_{2}$ with positive recoil (accelerating), and $n_{3}$ is the photon number. Note that the occupation number of the mode 1 is given by the sum of the other two, as a consequence of the constant of motion $C=n_{1}-n_{2}-n_{3}$, with $\langle C\rangle=0$ when 
the system starts from vacuum. For the state (31), the number variance is $\sigma_{i}^{2}=\left\langle n_{i}\right\rangle\left(1+\left\langle n_{i}\right\rangle\right)$ [1,14], i.e., the statistics is that of a thermal state. The state (31) is threemode entangled, i.e., the recoiling electrons and the emitted photons are entangled.

It can be seen that for $\bar{\rho} \leq 1$ the electrons, initially in the momentum state $n=0$, can populate only the lower momentum state $n=-1$, recoiling backward by $\hbar k$ when a photon is emitted [16]. In this quantum regime the system behaves as a two-level system [10], described by the two operators $c_{0}$ and $c_{-1}$. In the linear regime, the average number of photons grows exponentially as $\left\langle n_{3}\right\rangle \approx\left\langle n_{1}\right\rangle \approx$ $(1 / 4) \exp (\sqrt{\bar{\rho}} \bar{z})$ at resonance [i.e. for $\delta=1 /(2 \bar{\rho})]$ and the maximum number of emitted photons is $N$. In the quantum limit $\bar{\rho} \leq 1$, the state (31) reduces to the pure bipartite state with $\left\langle n_{2}\right\rangle \approx 0$ :

$$
|\Psi(\bar{z})\rangle \approx \frac{1}{\sqrt{1+\left\langle n_{1}\right\rangle}} \sum_{m=0}^{\infty} \alpha_{1}^{m}(\bar{z})|m, 0, m\rangle,
$$

where $\left|\alpha_{1}\right|^{2}=\left\langle n_{1}\right\rangle /\left(1+\left\langle n_{1}\right\rangle\right)$. The state (32) is maximally entangled, since the photon and the recoiling electron are generated in pairs.

Finally, we comment on the implication of the uncertainty relations (18) and (19) in the quantum limit $\bar{\rho} \leq 1$, in which the Hilbert space is spanned by only the two eigenstates of the discrete momentum, separated by $\hbar k$. Assuming for simplicity the semiclassical approximation, in which $c_{n}$ are treated as classical functions, and calling $P_{1}=\left|c_{0}\right|^{2} / N$ and $P_{2}=\left|c_{-1}\right|^{2} / N$ the probabilities of an electron occupying the state with $n=0$ or $n=-1$ (with $P_{1}+P_{2}=1$ ), it is easy to show that the momentum spread in units of $\hbar k$ is $\Delta p=\sqrt{P_{1}\left(1-P_{1}\right)}$. Hence, the maximum spread is $\Delta p_{\max }=1 / 2$ and it occurs for $P_{1}=P_{2}=1 / 2$. The inequality (19) states that, for $\Delta p=1 / 2,|b|<$ $1 / \sqrt{2} \approx 0.71$. However, in the two-state approximation, using Eq. (30), the bunching is $|b|=\left|c_{-1}^{*} c_{0}\right| / N=$ $\sqrt{P_{1} P_{2}}=\Delta p$, so that the maximum bunching is also $1 / 2$, which is consistent with the limitation given by (19).

\section{QUANTUM PROPAGATION MODEL}

In this section we extend a previous quantum model [13] to include the effects of propagation or slippage [17], which are fundamental to SASE, by using a multiple scaling method already adopted in classical FEL theory [18]. This allows us to take into account the existence of two different spatial length scales: the variation of the electron distribution on the scale of the radiation wavelength (describing the bunching on the variable $\theta$ ) and the variation of the field envelope on the much longer scale of the cooperation length, described by $z_{1}=2 \rho \theta=(z-$ $\left.v_{r} t\right) / \beta_{r} L_{c}$, i.e., the electron coordinate along the bunch, in units of the cooperation length, $L_{c}=\frac{\lambda}{4 \pi \rho}[4,7]$. We approximate the field operator $\hat{\Psi}$ by a classical wave function and describe the electromagnetic field classically. In the approach of Ref. [13], the field operator $\hat{\Psi}$ is approximated by a classical wave function $\Psi \approx\langle\hat{\Psi}\rangle / \sqrt{N}$ and the electromagnetic field is described classically by the dimensionless radiation amplitude, $A=a / \sqrt{\bar{\rho}} \bar{N}$. Then, the propagation effects for a quantum free-electron laser (QFEL) are described by the following equations:

$$
\begin{aligned}
i \frac{\partial \Psi\left(\theta, z_{1}, \bar{z}\right)}{\partial \bar{z}}= & -\frac{1}{2 \bar{\rho}} \frac{\partial^{2}}{\partial \theta^{2}} \Psi\left(\theta, z_{1}, \bar{z}\right) \\
& -i \bar{\rho}\left[A\left(z_{1}, \bar{z}\right) e^{i \theta}-\text { c.c. }\right] \Psi\left(\theta, z_{1}, \bar{z}\right) \\
\frac{\partial A\left(z_{1}, \bar{z}\right)}{\partial \bar{z}}+\frac{\partial A\left(z_{1}, \bar{z}\right)}{\partial z_{1}}= & \int_{0}^{2 \pi} d \theta\left|\Psi\left(\theta, z_{1}, \bar{z}\right)\right|^{2} e^{-i \theta} \\
& +i \delta A\left(z_{1}, \bar{z}\right) .
\end{aligned}
$$

The dimensionless field amplitude $A$ is defined so that $\bar{\rho}|\bar{A}|^{2}$ is the ratio between the photon density and the electron density. From Eqs. (33) and (34), one can show that the dimensionless density profile

$$
I_{0}\left(z_{1}\right)=\int_{0}^{2 \pi}|\Psi|^{2} d \theta
$$

is independent of $\bar{z}$. This means that the spatial distribution of the particles does not change appreciably on the slow scale $z_{1}$ during the interaction with the radiation field. The QFEL equations (33) and (34) depend only on the quantum FEL parameter, $\bar{\rho}$.

The classical limit of QFEL can be explicitly shown as follows. Extending the domain of $\theta$ to $(-\infty,+\infty)$ and defining the Wigner function associated to the wave function $\Psi$ [19]

$$
\begin{aligned}
W\left(\theta, \bar{p}, z_{1}, \bar{z}\right)= & \frac{\bar{\rho}}{2 \pi} \int_{-\infty}^{+\infty} d \theta^{\prime} e^{-i \bar{\rho} \theta^{\prime} \bar{p}} \Psi\left(\theta-\frac{\theta^{\prime}}{2}, z_{1}, \bar{z}\right)^{*} \\
& \times \Psi\left(\theta+\frac{\theta^{\prime}}{2}, z_{1}, \bar{z}\right),
\end{aligned}
$$

it is possible to show that Eqs. (33) and (34) are equivalent to the following equations $[16,17]$ :

$$
\begin{aligned}
& \frac{\partial W\left(\theta, \bar{p}, z_{1}, \bar{z}\right)}{\partial \bar{z}}+\bar{p} \frac{\partial W\left(\theta, \bar{p}, z_{1}, \bar{z}\right)}{\partial \theta}-\bar{\rho}\left(A e^{i \theta}+A^{*} e^{-i \theta}\right) \\
& \times\left[W\left(\theta, \bar{p}+\frac{1}{2 \bar{\rho}}, z_{1}, \bar{z}\right)-W\left(\theta, \bar{p}-\frac{1}{2 \bar{\rho}}, z_{1}, \bar{z}\right)\right]=0 \\
& \frac{\partial A}{\partial \bar{z}}+\frac{\partial A}{\partial z_{1}}=\int_{-\infty}^{+\infty} d \bar{p} \int_{-\infty}^{+\infty} d \theta W\left(\theta, \bar{p}, z_{1}, \bar{z}\right) e^{-i \theta}+i \delta A
\end{aligned}
$$

where $\bar{p}=m c\left(\gamma-\gamma_{0}\right) / \hbar k \bar{\rho}$. The Wigner function $W$ is a quasiprobability distribution, defined such that its marginal distributions are 


$$
\begin{aligned}
& \int_{-\infty}^{+\infty} d \bar{p} W\left(\theta, \bar{p}, z_{1}, \bar{z}\right)=\left|\Psi\left(\theta, z_{1}, \bar{z}\right)\right|^{2} \\
& \int_{-\infty}^{+\infty} d \theta W\left(\theta, \bar{p}, z_{1}, \bar{z}\right)=\left|\tilde{\Psi}\left(\bar{p}, z_{1}, \bar{z}\right)\right|^{2},
\end{aligned}
$$

where $\tilde{\Psi}\left(\bar{p}, z_{1}, \bar{z}\right)$ is the Fourier transform on $\theta$ of the wave function $\Psi\left(\theta, z_{1}, \bar{z}\right)$. Note that the quantum momentum shift of the Wigner function, $1 /(2 \bar{\rho})$, in dimensional units, would be $\hbar k / 2$. In Eq. (37), the difference between the Wigner functions becomes $\frac{\partial W}{\partial \bar{p}}$ in the limit $\bar{\rho} \rightarrow \infty$. Hence, for large values of $\bar{\rho}$, Eq. (37), which is equivalent to Eq. (33), reduces to the classical Vlasov equation:

$$
\begin{aligned}
\frac{\partial W\left(\theta, \bar{p}, z_{1}, \bar{z}\right)}{\partial \bar{z}} & +\bar{p} \frac{\partial W\left(\theta, \bar{p}, z_{1}, \bar{z}\right)}{\partial \theta} \\
& -\left(A e^{i \theta}+A^{*} e^{-i \theta}\right) \frac{\partial W\left(\theta, \bar{p}, z_{1}, \bar{z}\right)}{\partial \bar{p}}=0 .
\end{aligned}
$$

Equations (37) and (38) provide a description of the QFEL model in terms of the Wigner function, whereas Eqs. (38) and (39) are equivalent to the classical FEL model. Note that Eqs. (38) and (39) do not depend explicitly on $\bar{\rho}$, as must be the case in the classical model with universal scaling [3]. We briefly mention that Eq. (37) for the Wigner function has a broader validity than the Schrödinger equation (33), because it can also describe a statistical mixture of states which cannot be represented by a wave function but rather by a density operator.

Equations (33) and (34) are conveniently solved in the momentum representation. Assuming that $\Psi\left(\theta, z_{1}, \bar{z}\right)$ is a periodic function of $\theta$, it can be written as a Fourier series of momentum eigenstates $e^{i n \theta}$ as in (27):

$$
\Psi\left(\theta, z_{1}, \bar{z}\right)=\frac{1}{\sqrt{2 \pi}} \sum_{n=-\infty}^{\infty} c_{n}\left(z_{1}, \bar{z}\right) e^{i n(\theta+\delta \bar{z})} .
$$

where now $\left|c_{n}\left(\bar{z}, z_{1}\right)\right|^{2}$ is the local probability to have an electron with momentum $p=n(\hbar k)$ at $\bar{z}$ and $z_{1}$. So inserting Eq. (40) into Eqs. (33) and (34) and defining $A=$ $\bar{A} e^{i \delta \bar{z}}$, we obtain $[10,17]$

$$
\begin{gathered}
\frac{\partial c_{n}}{\partial \bar{z}}=-i E_{n} c_{n}-\bar{\rho}\left(\bar{A} c_{n-1}-\bar{A}^{*} c_{n+1}\right) \\
\frac{\partial \bar{A}}{\partial \bar{z}}+\frac{\partial \bar{A}}{\partial z_{1}}=\sum_{n=-\infty}^{\infty} c_{n} c_{n-1}^{*},
\end{gathered}
$$

where $E_{n}=\frac{n^{2}}{2 \bar{\rho}}+n \delta$. Equations (41) and (42) are the discrete QFEL model. They are our working equations and their numerical analysis will be discussed in the following section.

\section{A. Linear analysis}

We now perform a stability analysis of Eqs. (41) and (42) when the electrons initially occupy an arbitrary momentum eigenstate with momentum $n(\hbar k)$. As in sec. II, we assume that the system is in an equilibrium state with no field, $A=0$, and all the electrons in the state $n$, with $c_{n}=$ 1 and $c_{m}=0$ for all $m \neq n$. Looking for solutions of the linearized equations proportional to $e^{i\left(\lambda \bar{z}+\bar{\omega} z_{1}\right)}$ (where $\bar{\omega}=$ $\left(L_{c} / c\right)\left(\omega^{\prime}-\omega\right)=\left(\omega^{\prime}-\omega\right) /(2 \rho \omega)$ is the frequency shift of the radiation field with respect to the carrier frequency $\omega)$, one obtains the quantum dispersion relation

$$
\left(\lambda-\Delta_{n}\right)\left(\lambda^{2}-\frac{1}{4 \bar{\rho}^{2}}\right)+1=0
$$

where $\Delta_{n}=\delta+(n / \bar{\rho})-\bar{\omega}$. Note that the dispersion relation in Eq. (43) reduces to that in Eq. (13) when $n=0$ and $\bar{\omega}=0$. The behavior of the imaginary part of $\lambda$ as a function of $\Delta_{n}$ is the same as that shown in Fig. 1, with $\Delta_{n}$ instead of $\delta$. We recall that, when $\bar{\rho}<1$, the resonance moves from $\Delta_{n}=0$ to $\Delta_{n}=1 /(2 \bar{\rho})$, with a width of $4 \sqrt{\bar{\rho}}$ in units of $\Delta_{n}$. This corresponds, in the momentum space, to a shift of $\hbar k / 2$ with a width $4 \bar{\rho}^{3 / 2}(\hbar k)$. Let us now consider a fixed value of $\bar{\rho}$ and $\operatorname{plot} \operatorname{Im}(\lambda)$ for $\delta=0$ as a function of frequency shift $\bar{\omega}$, as shown in Fig. 2. It can be seen that the regions of the spectrum corresponding to gain $(\operatorname{Im}(\lambda)>0)$ appear as a series of discrete lines corresponding to different values of $n$. Each of these lines is centered on $\bar{\omega}=(2 n-1) /(2 \bar{\rho})$, equally separated by a distance $1 / \bar{\rho}$, and has a width of $4 \sqrt{\bar{\rho}}$. The transition to the classical limit of a broad, continuous gain spectrum can be seen from Fig. 2 to occur when the line separation becomes

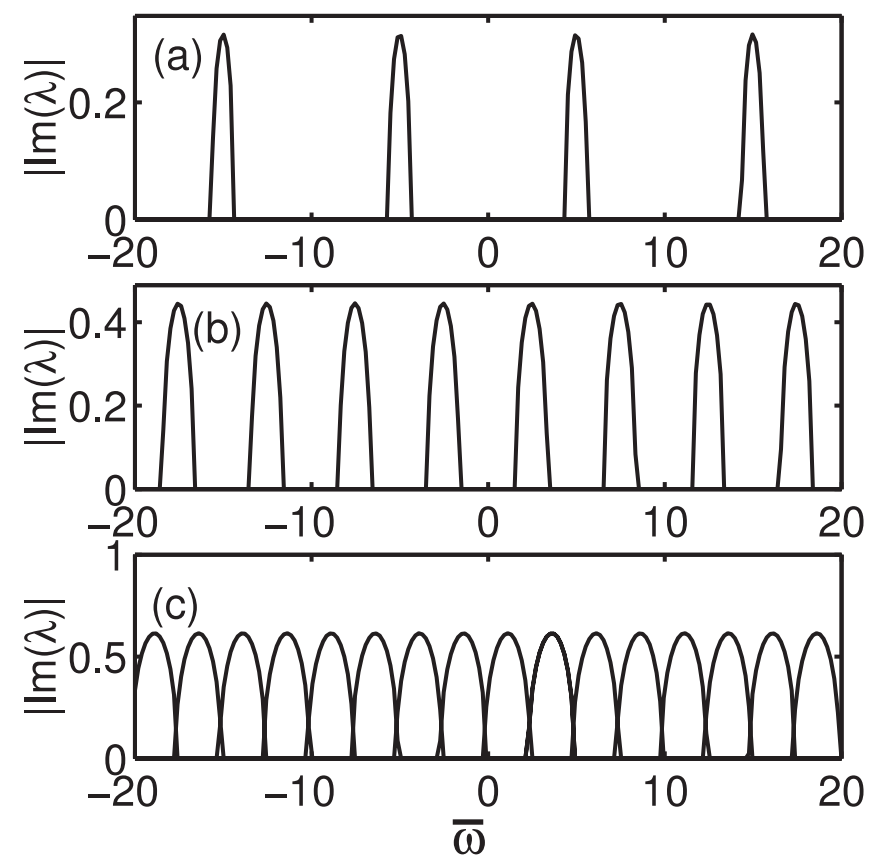

FIG. 2. Imaginary part of the unstable root of the cubic Eq. (43) vs $\bar{\omega}=\left(\omega^{\prime}-\omega\right) /(2 \rho \omega)$ for $\delta=0$, (a) $\bar{\rho}=0.1$, (b) $\bar{\rho}=0.2$, and (c) $\bar{\rho}=0.4$. Each line is centered around $\bar{\omega}=$ $(2 n-1) /(2 \bar{\rho})$ and has a width $4 \sqrt{\bar{\rho}}$. For $\bar{\rho}>0.4$, the lines overlap and the spectrum becomes continuous. 
smaller than the linewidth, i.e. $4 \bar{\rho}^{3 / 2}>1$ or $\bar{\rho}>$ $(1 / 2)^{4 / 3} \approx 0.4$.

The physical reason for these discrete frequencies is that in the quantum regime the electron recoils by $\hbar k$, so that electrons undergo a transition from an energy $E_{n} \propto p^{2} \propto$ $n^{2}$, to the state with energy $E_{n-1} \propto(n-1)^{2}$. Hence, the transition frequency varies as $1-2 n$, as shown above. As discussed in [15], for $\bar{\rho} \gg 1$ the electrons have almost the same probability of transition from the momentum state $n$ to the momentum states $n \pm 1$ (i.e. $\left|c_{n+1}\right|^{2} \approx\left|c_{n-1}\right|^{2}$ ), absorbing or emitting a photon. On the contrary, in the case $\bar{\rho}<1,\left|c_{n+1}\right|^{2} \ll\left|c_{n-1}\right|^{2}$, i.e., the particles can only emit a photon with transition $n \rightarrow n-1$, behaving approximately as a two- level system [10] described by the Maxwell-Bloch equations [20].

\section{B. Numerical results}

We now show that the discrete gain spectrum of the quantum regime shown in Fig. 2, can give rise to "quantum purification" of the SASE spectrum. Figure 3 shows a numerical simulation of the QFEL model Eqs. (41) and (42) for $L_{b}=30 L_{c}$ and $\delta=0$. The simulation assumes all electrons are initially in the momentum state $n=0$. The initial conditions for all the simulations are therefore $A\left(z_{1}, \bar{z}=0\right)=0, \quad c_{-1}\left(z_{1}, \bar{z}=0\right)=b_{0} e^{i \phi\left(z_{1}\right)}, \quad$ and $c_{0}\left(z_{1}, \bar{z}=0\right)=\sqrt{1-b_{0}^{2}}$, where $b_{0}=0.01$ and $\phi\left(z_{1}\right)$ is a randomly fluctuating phase with values in the range $[0,2 \pi)$. Figures $3(a)$ and $3(b)$ show the field intensity as a function of $z_{1}$ at $\bar{z}=30$ for the classical regime and $\bar{z}=$ 150 for the quantum regime, respectively. Figures 3(c) and 3 (d) show the corresponding classical and quantum power spectra of the radiated field versus $\bar{\omega}=\frac{\omega^{\prime}-\omega}{2 \rho \omega}$, where $\omega$ is the carrier frequency. It can be seen that there is a dramatic difference between the classical evolution [Figs. 3(a) and 3(c)] and the quantum evolution [Figs. 3(b) and 3(d)]. The temporal structure in the classical limit [Fig. 3(a)] is almost chaotic, with a broad spectrum. In contrast, the temporal behavior in the quantum limit [Fig. 3(b)] shows a purification of the initially noisy evolution, and the corresponding spectrum is composed by narrow lines, the positions of
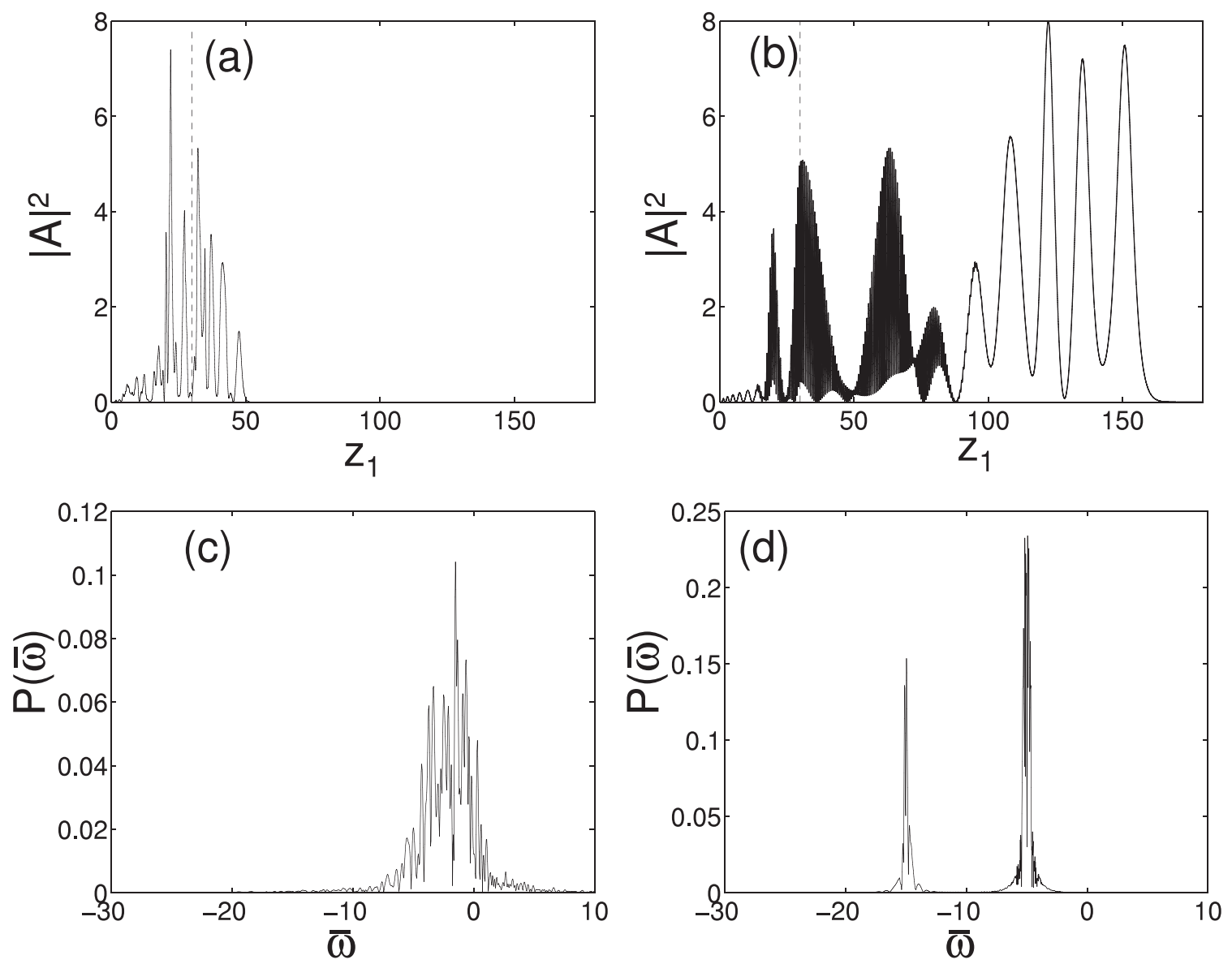

FIG. 3. Numerical solutions of Eqs. (41) and (42), for $L_{b}=30 L_{c}$ and $\delta=0$, in the classical regime $(\bar{\rho}=5$ and $\bar{z}=30)(\mathrm{a}),(\mathrm{c})$ and in the quantum regime $(\bar{\rho}=0.1$ and $\bar{z}=150)(\mathrm{b}),(\mathrm{d})$ : Graphs (a) and (b) show the scaled intensity and graphs (c) and (d) show the corresponding scaled power spectra as a function of scaled frequency $\bar{\omega}=\frac{\omega^{\prime}-\omega}{2 \rho \omega}$, where $\omega$ is the resonance frequency. The dotted line in (a) and (b) marks the front edge of the electron pulse. The frequency shift in (d) is in agreement with that predicted from Fig. 2(a). 

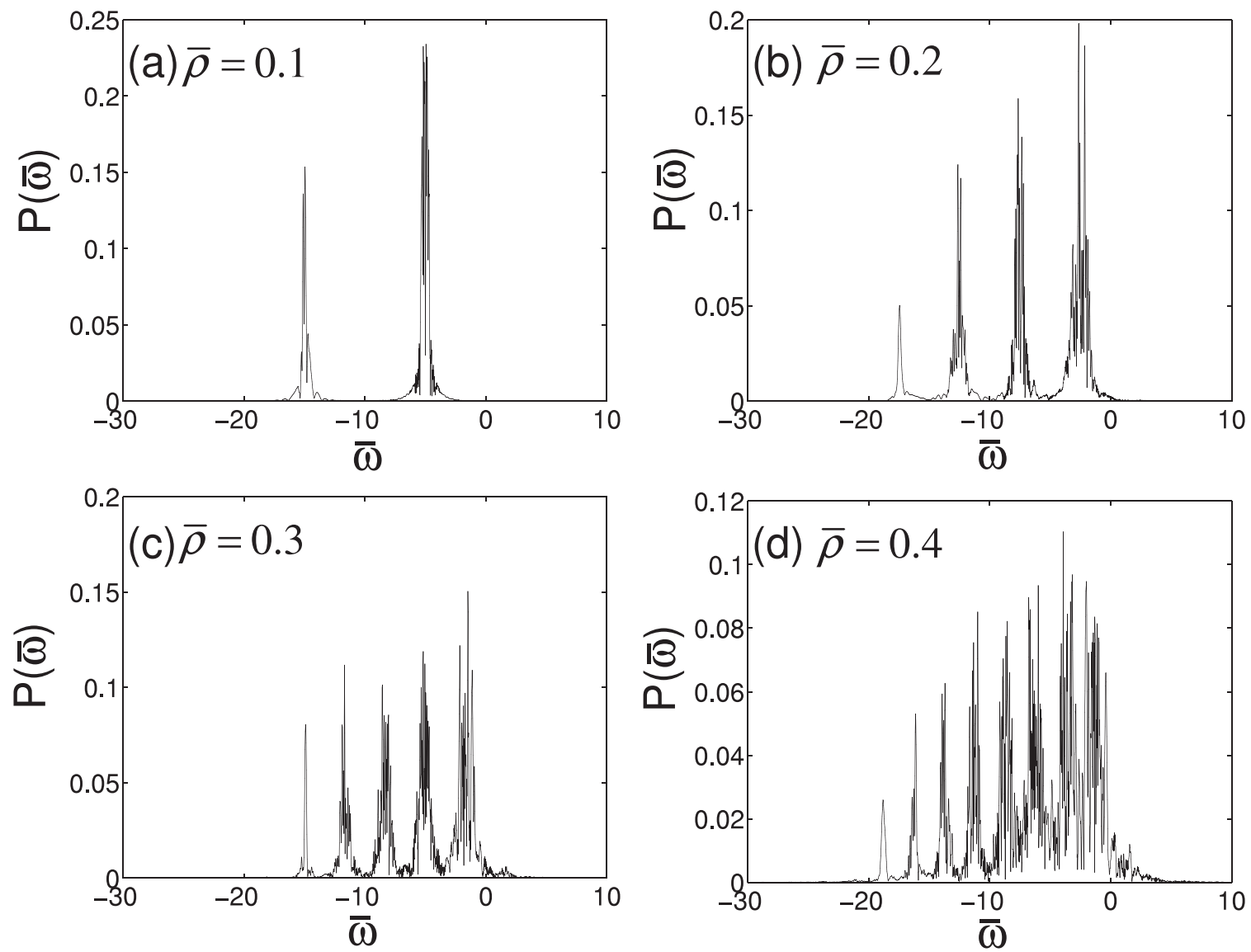

FIG. 4. The transition from quantum SASE to classical SASE: Scaled power spectra, $P(\bar{\omega})$, as a function of scaled frequency $\bar{\omega}=$ $\frac{\omega^{\prime}-\omega}{2 \rho \omega}$ for $\bar{z}=150$, calculated from a numerical solution of Eqs. (41) and (42) for $\delta=0$ when (a) $\bar{\rho}=0.1$, (b) $\bar{\rho}=0.2$, (c) $\bar{\rho}=0.3$, and (d) $\bar{\rho}=0.4$.

which are in agreement with that predicted by linear theory [see Fig. 2(a)]. Note that the line separation $1 / \bar{\rho}$ corresponds in real units to the relativistic recoil frequency $\frac{2 \hbar k^{2}}{\gamma_{r} m}$. The left-hand side of Fig. 3(b) is the rapid beat between the two frequencies of Fig. 3(d). For small values of $\bar{z}$ only the frequency with $\bar{\omega}=-1 /(2 \bar{\rho})$ appears. Increasing $\bar{z}$ additional lines downshifted by $1 / \bar{\rho}$ also appear.

The transition from the quantum regime of SASE to the classical regime is demonstrated in Fig. 4, which shows the scaled power spectra, $P(\bar{\omega})$, for different values of $\bar{\rho}$ calculated using Eqs. (41) and (42) for $\bar{z}=150$. It can be seen that there is a transition from discrete, narrow lines to a quasicontinuous spectrum when $\bar{\rho} \geq 0.4$, in agreement with the predictions of the linear analysis described in the previous section.

The reason for quantum purification of the SASE spectrum is as follows: As remarked earlier, in Figs. 1 and 2, the gain bandwidth decreases as $\sqrt{\bar{\rho}}$ and the cooperation length is longer by a factor $\sqrt{\bar{\rho}}$. Hence, one can understand that in quantum SASE, $\bar{\rho} \ll 1$, the system radiates coherently as if the start-up of the FEL interaction is initiated by a coherent bunching or a coherent seed.

\section{CONCLUSIONS}

In conclusion, we have studied two aspects of the quantum regime of a free-electron laser (QFEL). First, we revised the quantum linear theory of the $N$-particle freeelectron laser, introducing properly symmetrized electron collective operators. The correct cubic characteristic equation was then obtained, showing the shift and the narrowing of the FEL resonance. Our results demonstrate that the intrinsic quantum mechanical properties of the momentum and position operators imply a very general minimum uncertainty relation between energy spread and bunching, yielding a quantum limitation to the maximum bunching which can be obtained in an FEL. A minimum uncertainty state was properly defined so that it reduces to a Gaussian packet in the small fluctuation limit. Using a secondquantized treatment we have shown that, in the quantum regime, $\bar{\rho}<1$, the photon field and recoiling electrons are described by an maximally entangled quantum state. This property is well known to be quite fundamental for quantum information and quantum computing.

In the second part of our study, we have demonstrated the principle of the novel regime of quantum SASE, with 
dynamical properties very different from the usual classical SASE. In contrast to the classical limit, where the FEL dynamics are independent of $\bar{\rho}$, in the quantum limit where $\bar{\rho} \leq 1$, one has strong quantum effects and the FEL dynamics depend explicitly on $\bar{\rho}$. In particular, in the quantum SASE regime quantum purification of the temporal structure and of the spectrum occurs. The spectrum becomes a series of discrete narrow lines, separated in momentum space by $\hbar k$ and with a width $4 \bar{\rho}^{3 / 2}(\hbar k)$. The continuous and broad spectrum observed in classical SASE is recovered when $\bar{\rho}$ increases such that the width of each discrete line exceeds the separation between the lines, so that they overlap. The transition from the quantum to the classical SASE occurs for $\bar{\rho}>0.4$.

The possibility of experimental observation of this quantum regime has been envisaged in [21] using a laser wiggler and is currently under investigation [22].

\section{ACKNOWLEDGMENTS}

This work has been supported by INFN under the QFEL project. G. R. M.R. also acknowledges support from the Leverhulme Trust.

[1] R. Bonifacio and F. Casagrande, Opt. Commun. 50, 251 (1984); Nucl. Instrum. Methods Phys. Res., Sect. A 237, 168 (1985).

[2] C. B. Schroeder, C. Pellegrini, and P. Chen, Phys. Rev. E 64, 056502 (2001).

[3] R. Bonifacio, C. Pellegrini, and L. Narducci, Opt. Commun. 50, 373 1984).

[4] R. Bonifacio, L. De Salvo, P. Pierini, N. Piovella, and
C. Pellegrini, Phys. Rev. Lett. 73, 70 (1994).

[5] K. J. Kim, Phys. Rev. Lett. 57, 1871 (1986).

[6] J. M. Wang and L. H. Yu, Nucl. Instrum. Methods Phys. Res., Sect. A 250, 484 (1986).

[7] R. Bonifacio, B. W. J. McNeil, and P. Pierini, Phys. Rev. A 40, 4467 (1989).

[8] R. Bonifacio, C. Maroli, and N. Piovella, Opt. Commun. 68, 369 (1988).

[9] R. Bonifacio, F. Casagrande, G. Cerchioni, L. De Salvo Souza, P. Pierini, and N. Piovella, Riv. Nuovo Cimento 13, 9 (1990).

[10] R. Bonifacio, N. Piovella, and G. R. M. Robb, Nucl. Instrum. Methods Phys. Res., Sect. A 543, 645 (2005).

[11] P. Carruthers and M. M. Nieto, Rev. Mod. Phys. 40, 411 (1968).

[12] R. Jackiw, J. Math. Phys. (N.Y.) 9, 339 (1968).

[13] G. Preparata, Phys. Rev. A 38, 233 (1988).

[14] N. Piovella, M. Cola, and R. Bonifacio, Phys. Rev. A 67, 013817 (2003).

[15] N. Piovella, M. Gatelli, and R. Bonifacio, Opt. Commun. 194, 167 (2001).

[16] R. Bonifacio, M. M. Cola, N. Piovella, and G. R. M. Robb, Europhys. Lett. 69, 55 (2005).

[17] R. Bonifacio, N. Piovella, G. R. M. Robb, and M. M. Cola, Opt. Commun. 252, 381 (2005).

[18] G. T. Moore and M. O. Scully, Phys. Rev. A 21, 2000 (1980).

[19] M. Hillery, R. F. O’Connel, M. O. Scully, and E. P. Wigner, Phys. Rep. 106, 121 (1984).

[20] F. T. Arecchi, and R. Bonifacio, IEEE J. Quantum Electron. 1, 169 (1965).

[21] R. Bonifacio, Nucl. Instrum. Methods Phys. Res., Sect. A 546, 634 (2005)

[22] R. Bonifacio, M. Ferrario, N. Piovella, L. Serafini, and G. R. M. Robb, Proceedings of the 27th International Free Electron Laser Conference (Stanford, CA), 2005. 